Urol. Prax. 2020 $22: 153-159$

https://doi.org/10.1007/s41973-020-00118-7

Publié en ligne: 16 . Novembre 2020

(c) The Author(s) 2020

Riccardo Villani ${ }^{1}$ - Astrid Roosendaal' • Pauline Hämmerli ${ }^{2}$ Christophe E. Iselin

' Département de chirurgie, service d'Urologie HUG, Genève, Suisse

${ }^{2}$ Université de Genève, Genève, Suisse

\title{
PSA et IRM: comment s'en servir de façon raisonnable pour la détection du cancer de la prostate
}

1970 par Richard J. Ablin. Etudié avec précision, puis validé, par la Food and Drug Administration (FDA) en 1986, le PSA est largement utilisé dans le monde entier pour détecter et suivre le CaP. Il a ainsi révolutionné la prise en charge de cette maladie. Son introduction a permis de diagnostiquer des tumeurs aux stades plus précoces et ainsi de pouvoir diminuer la mortalité liée à la maladie; par exemple le taux de maladies d'emblée métastatiques est passé de $19,8 \%$ en 1989 à 3,3\% en 1998 en Pologne après l'introduction de ce marqueur [2].

Une dizaine d'années après l'introduction du PSA dans la pratique clinique, deux études prospectives ont investigué l'opportunité d'un dépistage de masse pour le $\mathrm{CaP}$, avec des résultats contradictoires. Létude PLCO (Prostate Lung Colorectal and Ovarian cancer screening trial), débutée en 1996 en Amérique du Nord, a randomisé plus de 76.000 patients suivis pour au moins 10 ans. De façon surprenante, le taux de mortalité liée au $\mathrm{CaP}$ du groupe de patients dépistés s'est avéré semblable à celui du groupe n’ayant pas bénéficié de dépistage [4]. Létude ERSCP (European Randomised Study of screening for Prostate Cancer; 182.000 patients suivis pendant plus de 10 ans) montre par contre, déjà dans sa première publication, une diminution substantielle du taux de mortalité spécifique du CaP de $20 \%$ [5].

La divergence entre les deux études s'explique par un biais dans la méthodologie de l'étude PLCO: plus de $50 \%$ des patients dans le groupe contrôle avaient déjà bénéficié d'au moins un dépistage avant d'être inclus dans l'étude.

Face à cette controverse, l'U.S. Preventive Services Task Force a publié en 2012 la recommandation de ne plus pratiquer le dépistage précoce du $\mathrm{CaP}$ (auparavant non recommandé seulement après l'âge de 75 ans). Consécutivement, on a assisté à une augmentation du diagnostic des adénocarcinomes prostatiques de risque intermédiaire ou de haut-risque, ainsi que des adénocarcinomes d'emblée métastatiques. Cette évolution a abouti à la recommandation de proposer une détection précoce chez le sujet informé des conséquences de celui-ci. Parallèlement, de nombreuses méta-analyses ont soutenu les avantages de la détection précoce par le PSA, en mettant en évidence des bénéfices sur la mortalité liée à la maladie, surtout en présence d'une détection effectuée suffisamment précocement [6-8].

En particulier, la Cochrane Review, publiée en 2013, montre l'utilité du dépistage en termes de : augmentation des diagnostics, diagnostic de maladies localisées et survie liée à la maladie [9]. Enfin, le sous-groupe scandinave de létude ERSPC a démontré à 14 ans un bénéfice de survie spécifique notoire dans le groupe dépistage, apparenté à celui mis en évidence pour le cancer du sein dépisté par mammographie [10].

En conclusion, le dosage du PSA est un test très sensible pour la détection du $\mathrm{CaP}$, mais il manque de spécificité, d’où la nécessité d'un contexte clinique précis pour éviter un haut nombre de faux le dosage du PSA (prostate-specific antigen) sanguin, protéine découverte en 
positifs. Actuellement, les Guidelines de l'EAU (European Association of Urology) ne recommandent pas de dépistage systématique, mais une détection précoce chez des patients sélectionnés et bien informés [11].

\section{De la prévention primaire à la détection précoce}

\section{Prévention primaire}

Plusieurs substances ont été investiguées dans un potentiel rôle de facteur de risque pouvant favoriser le développement d'un CaP. Aucune corrélation n'a été trouvée avec certains aliments (e.g. viande, alcool, acides gras oméga-3, lycopène ou matières grasses), le syndrome métabolique, la vitamine $\mathrm{D}$ ou les inhibiteurs de la 5- $\alpha$-reductase [12].

Il existe plusieurs études qui mettent en évidence un taux diminué de mortalité chez des patients diabétiques traités par metformine et atteint de $\mathrm{CaP}$; bien que le mécanisme antitumoral ne soit pas bien identifié (probable effet inhibiteur du médicament sur les cellules tumorales), le protocole expérimental STAMPEDE, utilisé dans le monde entier, prévoit l'introduction d'un traitement soit par metformine soit avec des anti-androgènes de deuxième génération (e.g. enzalutamide) pour le $\mathrm{CaP}$ avancé [13].

Une étude américaine montre une incidence diminuée du $\mathrm{CaP}$ chez des patients avec plus de 21 masturbations/éjaculations par mois par rapport à des patients avec 4-7 rapports sexuels par mois [14].

Cette donnée, si mise en corrélation avec une augmentation de l'incidence de $\mathrm{CaP}$ chez les patients qui ont fait une vasectomie, peut suggérer un rôle probable de la stagnation du liquide spermatique (inflammation locale ?) sur le développement d'un CaP.

Finalement le tabac, le travail nocturne, l'exposition au cadmium et la gonorrhée sont des facteurs en cours d'investigation, avec plusieurs articles qui évoquent un possible rôle en tant que facteurs de risque pour le $\mathrm{CaP}$; les phyto-oestrogènes semblent par contre avoir un rôle préventif.
Etant donné que le CaP est une tumeur hormono-sensible, il est important aussi de noter que l'administration de testostérone chez des patients avec un hypogonadisme naugmente pas le risque de $\mathrm{CaP}$ [15].

\section{Prévention secondaire}

Le dosage sanguin du PSA en est la pierre angulaire du screening. Toutefois son interprétation requiert des nuances particulières. En premier lieu, en cas de PSA élevé, il faut répéter un dosage quelques semaines plus tard pour écarter tout impact du rythme circadien ainsi qu'un biais du laboratoire. Deuxièmement, il faut s'assurer que le PSA ait été effectué en absence de facteurs confondants, tels qu'une infection urinaire, une stimulation prostatique mécanique (par exemple le toucher rectal [TR], échographie transrectale ou cystoscopie), un rapport sexuel, un tour en vélo ; toutes ces conditions peuvent provoquer une augmentation $\mathrm{du}$ PSA. Par ailleurs, il ne faut pas oublier que le PSA est un marqueur spécifique d’organe mais pas de maladie. Il peut également être augmenté en présence d'une importante hypertrophie bénigne de la prostate et/ou une prostatite chronique.

Il existe plusieurs paramètres qui peuvent aider à préciser la potentielle "significativité cancéreuse" du PSA. Plusieurs études montrent que la valeur initiale du PSA peut déjà classifier les patients en groupes de bas ou haut risque de présenter un CaP. Le Malmo Preventive Project (suivi de 1.167 patients durant 25 ans) montre que la plupart des décès liés au $\mathrm{CaP}(>90 \%)$ survient chez les patients avec un PSA $>2 \mathrm{ng} / \mathrm{ml}$ à l'âge de 60 ans [16]. L'étude ERSPC montre d'ailleurs qu'avec un PSA initial $<1 \mathrm{ng} / \mathrm{ml}$, le risque de présenter un carcinome de la prostate (PCa) est proche de $1 \%$ [17]. Par contre, les hommes avec un PSA $>1 \mathrm{ng} / \mathrm{ml}$ à 40 ans ou $>2 \mathrm{ng} / \mathrm{ml}$ à 60 ans ont un risque plus élevé de présenter une maladie plus agressive en termes de mortalité/morbidité dans les années à venir [18]. Par ailleurs, une intéressante étude montre qu'un seul dosage du PSA, effectué entre 50 et 69 ans, fait uniquement augmenter les diagnostics de $\mathrm{CaP}$ mais n'a aucune diminution significative sur la mortalité des patients [19], d'où la nécessité de mettre en place une stratégie de détection précoce à long terme.

Lorsque le PSA est autour de la limite supérieure de la norme $(4 \mathrm{ng} / \mathrm{ml})$, trois paramètres apportent certaines précisions.

La densité exprime le rapport entre PSA et le volume de la prostate; elle est utile pour différencier une augmentation du PSA lié à une hyperplasie prostatique significative.

Le ratio exprime le rapport entre PSA libre et PSA lié aux protéines. Inférieur à 0,10 , il en impose pour un risque plus élevé de CaP. Une étude effectuée chez 773 patients âgés de 50 à 75 ans avec un PSA entre 4 et $10 \mathrm{ng} / \mathrm{ml}$, démontre qu'en présence d'un ratio $>0,25$ le pourcentage de $\mathrm{CaP}$ retrouvé est de $8 \%$ contre $56 \%$ lorsque le ratio $<0,10$. Cependant le ratio n'est pas fiable pour un PSA $>10 \mathrm{ng} / \mathrm{ml}$ [20], reste non spécifique et doit être intégrée dans un contexte clinique précis.

La vélocité exprime la cinétique d'augmentation de la valeur du PSA dans le temps. Elle peut être évaluée comme valeur absolue d'augmentation annuelle du PSA (mesurée en $\mathrm{ng} / \mathrm{ml} / \mathrm{an}$ ) ou comme temps de doublement. Elle participe à la décision d'effectuer une biopsie si son augmentation est significative dans un court intervalle de temps. Ces trois paramètres restent malheureusement insuffisants à eux seuls pour poser l'indication à une biopsie.

Le TR est, au contraire du PSA, un test très spécifique mais avec une faible sensibilité ; combiné au dosage du PSA, il permet d'augmenter l'efficacité du dépistage. Il doit être effectué par le médecin de famille ou par l'urologue ; malheureusement, il nest pas garanti d'avoir une sensibilité optimale si le test est effectué par un médecin non expérimenté. Un TR permet normalement de diagnostiquer des tumeurs localisées dans la zone périphérique de la prostate (la majorité d'entre elles s'y situent), si le volume du nodule est $>0,2 \mathrm{ml}$. Un TR suspect a une valeur prédictive positive importante et est souvent en lien avec une maladie de haut grade ou localement invasive [21].

Face à toutes ces nuances, le contexte clinique et l'expérience sont incontournables pour éviter d'effectuer des biop- 
sies inutiles ou poser des diagnostics sans bénéfice sur l’espérance de vie ou la qualité de vie d'un patient. En résumé, les EAU Guidelines proposent d'adapter la stratégie de dépistage à la valeur du premier dosage du PSA : par exemple, un dosage aux 2 ans face à un PSA initialement déjà élevé, ou aux 5-6 ou voire 8-10 ans face à un PSA toujours bas (par exemple PSA $<1 \mathrm{ng} / \mathrm{ml}$ à 40 ans ou PSA $<2 \mathrm{ng} / \mathrm{ml}$ à 60 ans) [22]. Le taux de PSA doit être intégré par rapport à la présence de facteurs de risque avérés, qui sont l'histoire familiale de tumeur de la prostate ou la race noire. Notamment les hommes avec trois proches atteints ont un risque de CaP beaucoup plus élevé par rapportà des patients sans histoire familiale $(11,4 \%$ vs $1,4 \%)$. Les patients de race noire présentent plus souvent une maladie avancée et un taux de récidive plus élevé après prostatectomie radicale par rapport aux patients de race blanche ( $49 \%$ vs $26 \%$ ) [24]. Par ailleurs, les patients porteurs de la mutation génétique BCRA2 ont aussi un risque de $\mathrm{CaP}$ et de maladie de haut grade plus élevé [23].

Les Guidelines de l'EAU proposent donc d'adapter le dépistage par PSA pour ces populations à risque : la détection précoce doit être proposée dès l'âge de 50 ans pour la population générale, à lâge de 45 ans pour les patients avec une histoire familiale de $\mathrm{CaP}$ ou d'appartenance à la race noire, et à 40 ans en présence d'une mutation génétique BRCA2 (•Tab. 1).

L'EAU s'exprime aussi quant à la durée du dépistage : si celui-ci a été effectué correctement, il peut être arrêté à 75 ans. Pour les patients plus âgés, aucun dépistage systématique n’est préconisé.

Au plan pratique à ce jour, les trois outils initiaux de détection précoce du $\mathrm{CaP}$ sont le dosage du PSA sanguin total, la pratique d'un toucher rectal (TR) et la mesure du volume prostatique (afin détablir la densité du PSA). L'utilité combinée de ces trois données pour évaluer le risque individuel de $\mathrm{CaP}$ a été majorée par l'élaboration de divers nomogrammes (notamment ceux de l'ERSPC, disponible en ligne), qui peuvent être utilisés par le médecin de famille ou l'urologue traitant pour évaluer le risque de CaP. Selon l'importance du risque calculé, on propose une biopsie de la prostate (Risque
Urol. Prax. 2020 22:153-159 https://doi.org/10.1007/s41973-020-00118-7

(c) The Author(s) 2020

\section{R. Villani · A. Roosendaal · P. Hämmerli · C. E. Iselin \\ PSA et IRM: comment s'en servir de façon raisonnable pour la détection du cancer de la prostate}

\section{Résumé}

Le cancer de la prostate est la deuxième tumeur la plus fréquemment diagnostiquée chez les hommes. Elle est responsable d'un impact significatif sur la mortalité ou la qualité de vie des patients, mais, contrairement à d'autres tumeurs, est parfois une maladie à lente progression.

Une détection précoce avec dosage de I'antigène prostatique spécifique (PSA) et un toucher rectal doit être proposé à tout sujet masculin bien informé sur ses conséquences. Le double but du dépistage est de diagnostiquer les cancers potentiellement mortels ou causant une morbidité importante, tout évitant la mise en place d'investigations et traitements agressifs pour des maladies de bas grade, surtout chez les patients âgés.

Le PSA est un marqueur sensible mais non spécifique et son dosage doit être accompagné d'un contexte clinique précis, pour éviter la réalisation de biopsies superflues. L'imagerie par résonance magnétique multiparamétrique est un examen essentiel avant l'éventuelle réalisation d'une biopsie ainsi que pour la stadification et le suivi du cancer de la prostate (CaP).

Des nouveaux biomarqueurs plus spécifiques sont attendus de longue date pour améliorer la détection précoce du CaP.

\section{Mots clés}

Histoire familiale - Biopsie de prostate - Biomarqueurs · Score ERSPC . PI-RADS score

\section{PSA und MRT - wie man sie sinnvoll zur Früherkennung des Prostatakarzinoms nutzt}

\section{Zusammenfassung}

Das Prostatakarzinom ist die zweithäufigste Tumordiagnose bei Männern. Es hat wesentliche Auswirkungen auf die Mortalität und die Lebensqualität der Patienten, aber im Gegensatz zu anderen Tumoren ist das Fortschreiten der Erkrankung manchmal langsam.

Eine Früherkennung mit PSA-Bestimmung und MRT sollte jedem männlichen Patienten nach Aufklärung über die Auswirkungen angeboten werden.

Das zweifache Ziel der Früherkennung besteht darin, einerseits lebensbedrohliche oder mit hoher Morbidität einhergehende Krebserkrankungen zu diagnostizieren, aber andererseits - insbesondere bei älteren Patienten - aggressive Untersuchungs- und

$<12,5 \% \rightarrow$ pas de biopsie. Risque 12,5 à $20 \% \rightarrow$ considérer éventuellement une biopsie selon comorbidités, hérédité, etc. Risque $>20 \% \rightarrow$ biopsie).

Ces nomogrammes sont recommandés dans les Guidelines de l'EAU.

Létude prospective de l'ERSPC mentionnée ci-dessus a comparé différentes durées de dépistage, ce qui a permis de démontrer que plus celui-ci est long, plus il est efficace.
Therapieformen bei niedriggradigen Tumoren zu vermeiden.

Der PSA-Wert ist ein sensitiver, aber unspezifischer Marker. Seine Bestimmung muss unter Beachtung des genauen klinischen Zusammenhangs erfolgen, um unnötige Biopsien zu vermeiden.

Die multiparametrische MRT ist eine wesentliche Untersuchung vor der eventuellen Durchführung einer Biopsie sowie für das Staging und die Überwachung des PCa. Seit langem hofft man auf neue, spezifischere Biomarker für eine bessere Früherkennung des PCa.

Schlüsselwörter

Familienvorgeschichte - Prostatabiopsie . Biomarker · ERSPC-Score · PI-RADS-Score

Ainsi, le nombre de patients auxquels proposer un dépistage pour éviter un décès dû au CaP (NNS - number needed to screen) diminue drastiquement, permettant de dépasser le dépistage pour le carcinome mammaire en termes d'efficacité et de rapport coût/bénéfice. En termes concrets, le nombre de patients à inclure dans le dépistage pour éviter un décès est de 742 avec un dépistage de 13 ans et de 570 avec un dépistage 


\begin{tabular}{l} 
Tab. 1 Règles de l'EAU sur le dépistage du \\
CaP en fonction de l'âge et des facteurs de \\
risque \\
Dépistage précoce du carcinome de la \\
prostate (PCa) \\
\hline Hommes d'âge $>50$ ans \\
Hommes d'âge $>45$ ans en présence \\
d'histoire familière positive de PCa \\
Hommes d'âge $>45$ ans d'origine africaine \\
Hommes d'âge $>40$ ans porteurs d'une \\
mutation BRCA2
\end{tabular}

de 16 ans [24]. À titre comparatif, le nombre de patientes entre 50 et 59 ans auxquelles proposer une mammographie pour éviter un décès dû au cancer mammaire est de 1.340 .

Ces directives n'empêchent pas d'effectuer un dosage du PSA chez un patient de plus de 75 ans en parfait état général avec une espérance de vie de 15 ans. La règle suggère d'effectuer un dépistage chez un patient avec une espérance de vie de $>10-15$ ans, car poser un diagnostic de $\mathrm{CaP}$ chez un patient avec une espérance de vie restreinte n'aura pas d'impact sur la mortalité du patient. Au contraire, cela engendrera une augmentation du taux de surtraitement et, par conséquent, une augmentation du risque de complications possibles, et donc du taux de morbidité, ainsi que du coût sanitaire.

Cependant, l'investigation d'un patient plus âgé mais symptomatique avec une suspicion clinique de $\mathrm{CaP}$ reste une option valable, dans le but de mettre en place un traitement palliatif.

Typiquement, il est possible d'avoir des patients âgés qui deviennent symptomatiques sur un $\mathrm{CaP}$ avancé, par exemple:

- douleur lombaire (ou en général osseuse) en cas de tassement métastatique de la colonne vertébrale;

- rétention urinaire du fait de l'hypertrophie prostatique tumorale;

- dilatation pyélocalicielle (compliquée soit d'une insuffisance rénale, d'une douleur lombaire ou d'une pyélonéphrite) sur compression urétérale extrinsèque.

En présence d'une des complications susmentionnées, il est toujours possible de mettre en place un traitement palliatif, notamment un traitement systémique par
Tab. 2 Classification PI-RADS v2 (validée en 2019)

Catégories d'évaluation PI-RADS v2

\begin{tabular}{|l|l|}
\hline PI-RADS 1 & $\begin{array}{l}\text { Très faible (il est très peu pro- } \\
\text { bable qu'un cancer clinique- } \\
\text { ment significatif soit présent) }\end{array}$ \\
\hline PI-RADS 2 & $\begin{array}{l}\text { Faible (il est peu probable } \\
\text { qu'un cancer cliniquement } \\
\text { significatif soit présent) }\end{array}$ \\
\hline PI-RADS 3 & $\begin{array}{l}\text { Intermédiaire (la présence d'un } \\
\text { cancer cliniquement significatif } \\
\text { est équivoque, incertaine) }\end{array}$ \\
\hline PI-RADS 4 & $\begin{array}{l}\text { Élevé (la présence d'un cancer } \\
\text { est susceptible d'être clinique- } \\
\text { ment significatif) }\end{array}$ \\
\hline PI-RADS 5 & $\begin{array}{l}\text { Très élevé (un cancer clini- } \\
\text { quement significatif est très } \\
\text { probablement présent) }\end{array}$ \\
\hline
\end{tabular}

déprivation androgénique, couplé à un éventuel traitement local (e.g. radiothérapie ciblée sur les métastases, intervention désobstructive prostatique, mise en place d'un drainage rénal, etc.).

\section{Les nouveaux biomarqueurs}

De nouveaux biomarqueurs sont en cours d'investigation : 4-kallikrein-panel (4K) ou Prostate Health Index test (PHI) dans le bilan sanguin, recherche de m-RNA PCA3 (prostate cancer antigen) ou TMPRSS2-ERG (trans-membrane protease serine 2) dans les urines.

Plusieurs études montrent un possible rôle de ces marqueurs pour différencier des maladies de haut et bas grade; actuellement, à cause surtout du manque d'études et de financements, ces examens nont pas encore fait leurs preuves.

Actuellement, les Guidelines de l'EAU ne suggèrent d'utiliser ces nouveaux biomarqueurs (notamment le PHI) que pour le suivi d'un patient avec une précédente biopsie négative de prostate, en attente de nouvelles données.

\section{IRM multiparametrique}

Traditionnellement, l'IRM était limitée à la stadification du CaP, mais grâce aux techniques d'imagerie avancées, son utilisation s'est étendue à divers stades de la pratique clinique, notamment la détection des tumeurs et le suivi de la maladie pendant une surveillance active.
LIRM multiparamétrique de la prostate (IRMmp) est une modalité radiologique qui combine l'imagerie IRM anatomique avec une ou plusieurs séquences IRM fonctionnelles. C'est actuellement la modalité d'imagerie la plus précise pour détecter, localiser et déterminer le stade du cancer de la prostate. Elle fournit des informations anatomiques en utilisant l'imagerie pondérée en T2, ainsi que des informations métaboliques grâce aux techniques de contraste amélioré (IRM-DCE) et de diffusion (IDP) ([25]; • Fig. 1). Elle peut ainsi identifier différents types de lésion.

Le score Prostate Imaging Reporting and Data System (PI-RADS) a été introduit en 2012 pour les classifier. Cette classification a révolutionné l'imagerie de la prostate en standardisant la façon dont l'examen est acquis et interprété. Elle a été révisée et simplifiée en 2015 (PIRADS version 2) et continue d'évoluer (PI-RADS version 2.1 de 2019).

Le score PI-RADS v2 utilise une échelle de cinq points dépendants des résultats d'IRMmp sur les différentes phases d'acquisitions. Pour chaque lésion ce score définira le risque d'un cancer de prostate (• Tab. 2).

Actuellement, en cas de suspicion clinique (TR) ou biologique (PSA) de CaP, il n'est pas recommandé d'effectuer des biopsies randomisées de la prostate, mais les Guidelines de l'EAU recommandent d'effectuer d'emblée une IRMmp à la recherche d'un nodule prostatique sur lequel éventuellement cibler une biopsie.

La corrélation avec les échantillons des biopsies montre que l'IRMmp a une bonne sensibilité pour la détection et la localisation des cancers de grade intermédiaire et élevé.

Dans une récente méta-analyse Cochrane qui a comparé l'IRMmp à des biopsies modèles ( $>20$ carottes) dans des contextes de biopsies natives et répétées, l'IRMmp avait une sensibilité élevée (>90\%) et une spécificité de $35 \%$ (grade ISUP $>2$ ).

Elle est par contre moins sensible pour identifier les $\mathrm{CaP}$ de bas grade (International Society of Urological Pathology [ISUP] grade 1), avec une sensibilité globale de $70 \%$. 

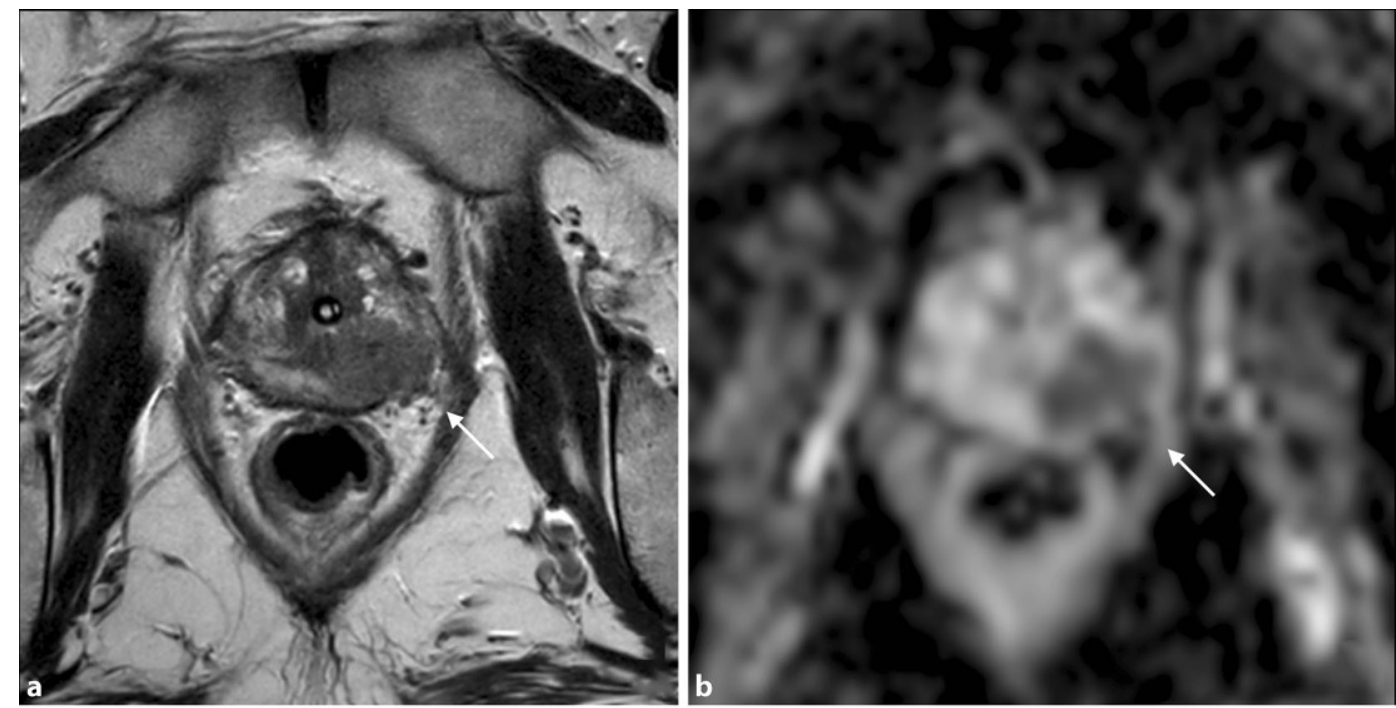

Fig. $1<$ L'IRM multiparamétrique (IRMmp) montrant une lésion prostatique de la zone périphérique postéro-médiane gauche avec effraction de la capsu-
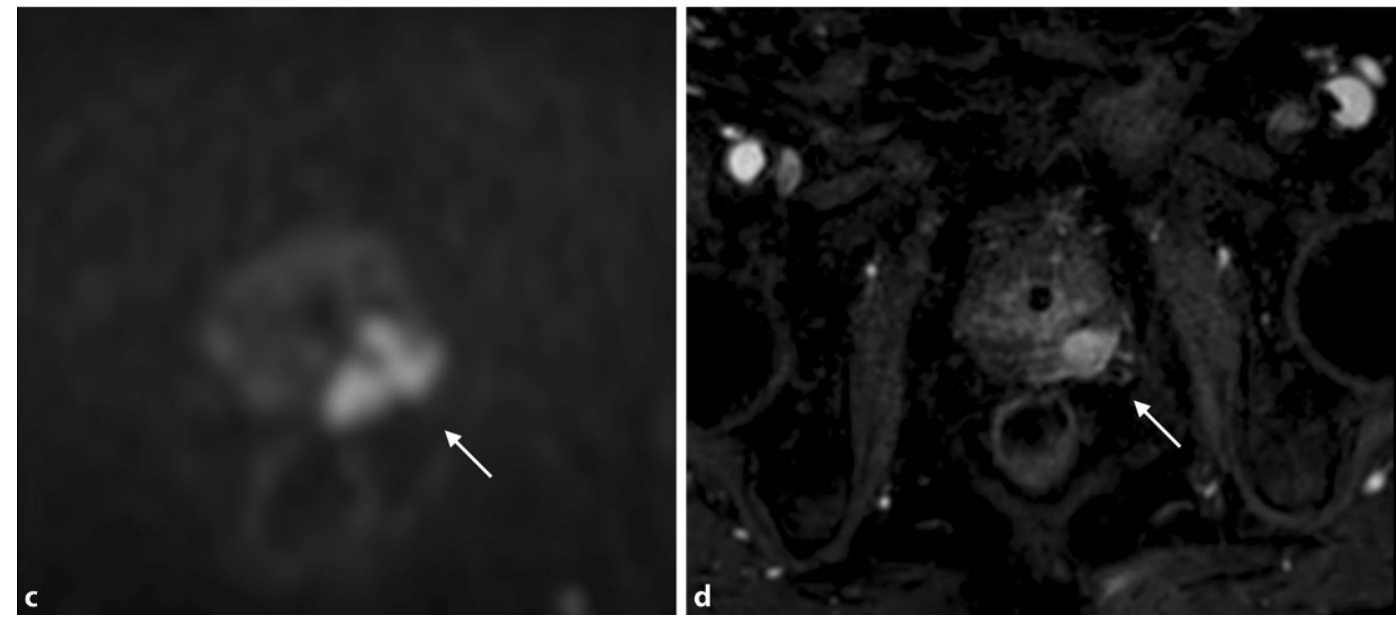
le prostatique, classé Prostate Imaging Reporting and Data System (PI-RADS) 5. a Séquence anatomique en pondération $\mathrm{T} 2 \mathrm{mon}$ trant la lésion sous forme d'une plage en hyposignal. b, c Séquences fonctionnelles de diffusion (ADC ethigh b-value DWI) avec hypointensité de signal en $A D C$ et hyperintensité en high b-valueDWI.dSéquence de perfusion dynamique (dynamic contrast enhancement - DCE), montrant un rehaussement intense et précoce de la lésion

Ainsi, en raison du manque de spécificité de l'IRMmp, on trouve parfois des maladies de bas grade, d'où la recommandation de ne pas effectuer d'IRMmp comme test de détection chez les patients sans suspicion clinique (TR ou PSA pathologique).

Grâce à l'IRMmp, le 30-60 \% des biopsies superflues sont aujourd'hui évitées.

En cas de suspicion clinique ou biologique de CaP, la découverte à l'IRMmp d'un nodule PI-RADS $>3$ pose normalement l'indication à effectuer une biopsie ciblée de ce nodule, étant donné un risque de $\mathrm{CaP}$ cliniquement significatif (ISUP $>1$ ) d'environ $10 \%$ avec une lésion PI-RADS 3, de $28 \%$ avec une lésion PIRADS 4 et de $54 \%$ avec une lésion PIRADS 5 [26].

Le score PI-RADS peut aussi donner une indication sur le grade de carcinome prostatique quon va éventuellement trouver à la biopsie : les lésions PI-RADS 4 et 5 sont normalement associées à des tumeurs ISUP $>2$.

Dans un avenir possiblement proche se pose la question futuriste de la détection primaire (sans s'aider du PSA) du $\mathrm{CaP}$ par IRM. Cependant jusqu'à ce jour, aucun travail n'a pu valider une telle perspective. Par contre, l'information apportée par l'IRM est actuellement déjà incorporée dans certains nomogrammes (e.g. ERSPC) stratifiant la probabilité de biopsie positive. Dans une perspective d'épargne financière, il y a actuellement un gros courant de recherche visant à prédire l'opportunité de la réalisation d'une IRM, puis d'une éventuelle biopsie au moyen de nomogrammes combinants TR, volume prostatique et PSA ou nouveaux marqueurs [27].

\section{Les biopsies ciblées de prostate}

Les biopsies prostatiques ciblées sur les images IRM sont désormais effectuées selon la technique de fusion. Cette méthode permet de superposer des images IRMmp avec des images échographiques tridimensionnelles (3D) lors de la pratique de la biopsie. Notre étude a montré en 2017 que cette technique a un taux de détection de $\mathrm{CaP}$ supérieur aux autres techniques ( $58 \%$, vs $38 \%$ sous échographie $3 \mathrm{D}$ seule et $19 \%$ sous échographie $2 \mathrm{D}$ seule) [28].

Le taux de détection augmente avec l'augmentation du grade ISUP. Les biopsies ciblées n'exposent pas à un risque de surtraitement car elles identifient moins de tumeurs de bas grade (ISUP 1), qui sont des tumeurs cliniquement peu significatives.

Les biopsies peuvent être effectuées par voie transrectale (TR) ou trans- 
périnéale (TP), sans différence entre les taux de détection; la biopsie transpérinéale est par contre associée à un moindre taux d'infections et de saignement (RR de 0,26 et de 0,79 entre biopsie TR et TP). Les biopsies TP sont ainsi particulièrement indiquées chez les patients diabétiques, sondés ou en général à risque de prostatite, avec une tendance à la généraliser à tous les patients en raison du risque infectieux moindre, et de la cartographie prostatique antérieure mieux échantillonnée que par voie TR. La biopsie TP peut être par contre associée à une symptomatologie algique les jours après le geste (RR 1,83) [29].

Des biopsies randomisées du reste de la prostate sont toujours effectuées en plus des biopsies ciblées en raison des $8 \%$ de tumeurs cliniquement significatives qui sont identifiées en dehors des nodules biopsies sous méthode fusion, ce qui permet évidemment de mieux planifier la stratégie de prise en charge (traitement focal ou radical, ou surveillance active).

\section{Conclusions}

Le cancer de la prostate, contrairement à d'autres tumeurs, est parfois une maladie à lente progression, mais très prévalente dans la population et capable de causer un taux élevé de morbidité et mortalité.

Une détection précoce avec dosage du PSA et TR devrait donc être proposé à tout sujet masculin bien informé sur ses conséquences, selon les recommandations indiquées dans cet article, conformément aux Guidelines de l'EAU.

Le double but du dépistage est de diagnostiquer les cancers potentiellement mortels ou causant une morbidité importante, tout évitant la mise en place d'investigations et traitements agressifs pour des maladies de bas grade, surtout chez les patients âgés.

Le PSA est un marqueur sensible mais spécifique seulement d'organe et pas de maladie.

L'interprétation de son résultat doit être accompagné d'un contexte clinique précis, pour éviter la réalisation de biopsies superflues. Les scores ERSPC sont très utiles pour orienter le médecin dans l'indication à une biopsie face à un PSA suspect.

L'IRM multiparamétrique est un examen essentiel avant l'éventuelle réalisation d'une biopsie ainsi que pour la stadification et le suivi du CaP.

Des nouveaux biomarqueurs plus spécifiques sont attendus de longue date pour améliorer la détection précoce du $\mathrm{CaP}$, mais sont toujours à l'heure actuelle au stade investigatif.

\section{Adresse de correspondance}

\section{Riccardo Villani}

Département de chirurgie, service d'Urologie HUG

rue Gabrielle-Perret Gentil 4, 1205 Genève,

Suisse

riccardo.villani@hcuge.ch

Funding. Open access funding provided by Univer sity of Geneva

\section{Conformité aux directives éthiques}

Conflit d'intérêts R. Villani, A. Roosendaal, P. Hämmerli et C.E. Iselin déclarent ne pas avoir de conflit d'intérêt.

Pour cet article, les auteurs n'ont réalisé aucune étude sur les humains ou les animaux. Les études réalisées étaient conformes aux normes éthiques indiquées dans chaque cas.

Open Access. This article is licensed under a Creative Commons Attribution 4.0 International License, which permits use, sharing, adaptation, distribution and reproduction in any medium or format, as long as you give appropriate credit to the original author(s) and the source, provide a link to the Creative Commons licence, and indicate if changes were made. The images or other third party material in this article are included in the article's Creative Commons licence, unless indicated otherwise in a credit line to the material. If material is not included in the article's Creative Commons licence and your intended use is not permitted by statutory regulation or exceeds the permitted use, you will need to obtain permission directly from the copyright holder. To view a copy of this licence, visit http://creativecommons.org/licenses/by/4.0/.

\section{Références}

1. Rocco B, Grasso A, Mottrie A et al (2012) PSA mass screening: is there enough evidence? Cent European JUrol 65(1):4-6

2. Oliver SE, May MT, Gunnell D (2001) International trends in prostate-cancer mortality in the "PSA ERA". Int J Cancer 92 (6):893-898

3. Wojciechowska U, Didkowska J, Zatoński W (2010) Nowotwory złośliwe w Polsce w 2008 roku
[Malignant neoplastic in Poland (2006)]. Centrum Onkologii Instytut, Warszawa, S40-41

4. Paul F, Pinsky PF, Miller E, Prorok P et al (2019) Extended follow-up for prostate cancer incidence and mortality among participants in the prostate, lung, colorectal and ovarian randomized cancer screening trial. BJU Int 123(5):854-860

5. Hugosson J, Roobol MJ, Mansson M et al (2019) A 16-yr follow-up of the European randomized study of screening for prostate cancer. Our Urol 76(1):43-51

6. La Rochelle I, Amling CL (2010) Prostate cancer screening: what have we learned from the PLCO and ERSPC trials. Curr Urol Rep 11(3):198-201

7. Eckersberger $E$ et al (2009) Screening for prostate cancer: a review of the ERSPC and PLCO trials. Rev Urol 11(3):127-133

8. Sandblom G et al (2011) Randomised prostate cancer screening trial: 20 year follow-up. BMJ 342:d1539

9. Ilic D et al (2013) Screening for prostate cancer. Cochrane Database Syst Rev. https://doi.org/10. 1002/14651858.CD004720.pub3

10. Hugosson I et al (2010) Mortality results from the Göteborg randomised population-based prostatecancer screening trial. Lancet Oncol 11:725-732

11. Arnsrud Godtman R et al (2015) Opportunistic testing versus organized prostate-specific antigen screening: outcome after 18 years in the Goteborg randomized population-based prostate cancer screening trial. Eur Urol 68:354

12. Leitzmann MF et al (2012) Risk factors for the onset of prostatic cancer: age, location, and behavioral correlates. Clin Epidemiol 4:1

13. Gillessen S, Gilson C, James N et al (2016) Repurposing Metfomin as therapy for prostate cancer within the STAMPEDE trial platform. Eur Urol 70(6):906-908

14. Rider JR et al (2016) Ejaculation frequency and risk of prostate cancer: updated results with an additional decade of follow-up. Eur Urol 70:974

15. Watts EL et al (2018) Low free testosterone and prostate cancer risk: a collaborative analysis of 20 prospective studies. Eur Urol 74:585

16. Carlsson S et al (2014) Influence of blood prostate specific antigen levels at age 60 on benefits and harms of prostate cancer screening: population based cohort study. BMJ 348:g2296

17. Roobol MJ et al (2005) Is additional testing necessary in men with prostate-specific antigen levels of $1.0 \mathrm{ng} / \mathrm{mL}$ or less in a population-based screening setting? (ERSPC, section Rotterdam). Urology 65:343

18. Vickers AJ et al (2013) Strategy for detection of prostate cancer based on relation between prostate specific antigen at age 40-55 and long term risk of metastasis: case-control study. BMJ 346:f2023

19. Martin RM etal (2018) Effect of a low-intensity PSAbased screening intervention on prostate cancer mortality: the CAP randomized clinical trial. JAMA 319:883

20. Catalona WJ et al (1998) Use of the percentage of free prostate-specific antigen to enhance differentiation of prostate cancer from benign prostatic disease: a prospective multicenter clinical trial. JAMA 279:1542

21. Okotie OT et al (2007) Characteristics of prostate cancer detected by digital rectal examination only. Urology 70:1117

22. Gelfond J et al (2015) Intermediate-term risk of prostate cancer is directly related to baseline prostate specific antigen:implications for reducing 
the burden of prostate specific antigen screening JUrol 194:46

23. Bratt $O$ et al (2016) Family history and probability of prostate cancer, differentiated by risk category: a nationwide population-based study. J Natl Cancer Inst. https://doi.org/10.1093/jnci/djw110

24. Hugosson J et al (2019) A 16-yr follow-up of the European randomized study of screening for prostate cancer. Eur Urol 76:43

25. Flutterer J et al (2017) Multiparametric MRI in the detection of clinically significant prostate cancer. Korean J Radiol 18(4):597-606

26. Zhang Y, Zhu G, Zhao W et al (2020) A PI-RADSbased new nomogram for predicting clinically significant prostate cancer: a cohort study. Cancer Manag Res 12:3631-3641

27. Osses DF, Roobol MJ, Schoots IG (2019) Prediction medicine: biomarkers, risk calculators and magnetic resonance imaging as risk stratification tools in prostate cancer diagnosis. Int J Mol Sci 20:1637-1656

28. Klein J, de Górski A, Iselin CEet al (2017) Transrectal Ultrasound-Guided Prostate Biopsy for Cancer Detection: Performance of 2D-, 3D- and 3D-MRI Fusion Targeted Techniques. Urol Int 98(1):7-14

29. Jianjian Xiang, Huaqing Y, Jiangfeng L et al (2019) Transperineal versus transrectal prostate biopsy in the diagnosis of prostate cancer: a systematic reviewand meta-analysis. World JSurg Oncol 17:31

Note de l'éditeur. Springer Nature conserve une position neutre en ce qui concerne les revendications juridictionnelles dans les cartes géographiques et les affiliations institutionnelles figurant dans ses publications.
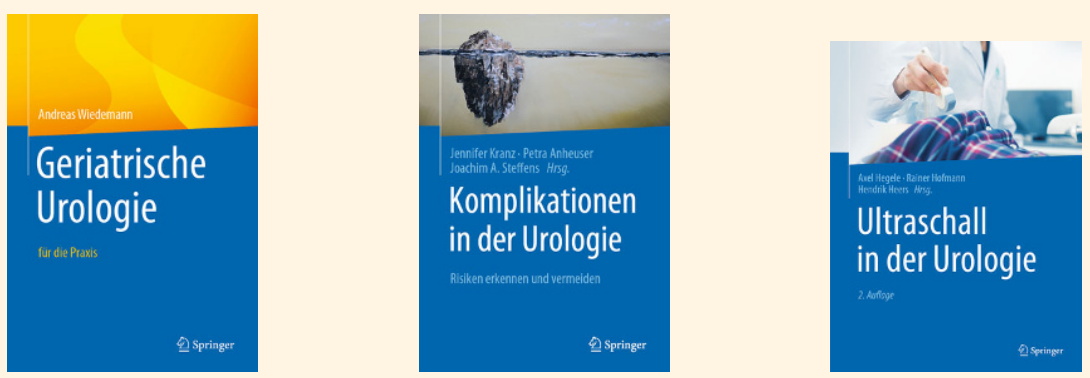

\section{Aktuelle Buchempfehlungen in der Urologie}

\section{Geriatrische Urologie - für die Praxis}

A. Wiedemann (Hrsg.)

1. Aufl. 2020,237 S. Softcover $54,99 €$, eBook $42,99 €$

Tipps für eine abgestimmte Behandlung von geriatrisch urologischen Patienten

Das Praxisbuch zur Urogeriatrie. Als erster Professor für Urogeriatrie Deutschlands schafft der Autor den Transfer von hilfreichen Erkenntnissen aus der Geriatrie in die Urologie und umgekehrt. Die Zunahme älterer, multimorbider Patienten ist in der Urologie besonders stark. Inkontinenz ist eines der führenden Leiden im höheren Lebensalter. Fachübergreifend, auf der Basis der aktuellen Wissenschaft und einer jahrelangen Erfahrung aus Klinikpraxis und Forschung, werden relevante Aspekte der Geriatrie, die bei der Behandlung multimorbider, urologischer Patienten berücksichtigt werden sollten, übersichtlich und praxisnah erläutert.

\section{Komplikationen in der Urologie - Risiken erkennen und vermeiden}

J. Kranz, P. Anheuser, J.A. Steffens (Hrsg.),

1. Aufl. 2020, 478 S. Softcover $79,99 €$, eBook $62,99 €$

Erscheinungstermin: 10. Dezember 2020

Dieses Buch schildert Komplikationen und Zwischenfälle aus dem gesamten Fachbereich der Urologie. Neben konservativen und operativen Therapieoptionen werden auch die Indikationsstellung verschiedener Verfahren sowie diagnostische Maßnahmen einbezogen. Unter Berücksichtigung des aktuellen Wissenstandes bietet das Buch Strategien zur Vermeidung und Therapievorschläge zur Beherrschung von Komplikationen. Häufigkeitsangaben typischer, aber auch seltener Komplikationen werden systematisch aufgeführt. Der urologisch tätige Arzt erhält Tipps für ein strukturiertes Risikomanagement.Für alle operativ tätigen Urologen und niedergelassenen Fachärzte, aber auch zur Erstellung von Gutachten, dient es als wertvolle Lektüre.

\section{Ultraschall in der Urologie}

A. Hegele, R. Hofmann, H. Heers (Hrsg.)

2. Aufl. 2020, 339 S. Hardcover $129,99 €$, eBook $99,99 €$

Erscheinungstermin: 11. Dezember 2020

Der Atlas „Ultraschall in der Urologie“ ist Lehrbuch und Nachschlagewerk zugleich. Er liefert dem klinisch tätigen Urologen einen reichen Fundus von über 400 Referenzaufnahmen häufiger Befunde und interessanter Raritäten. Neben technischen Grundlagen der Sonographie sowie praktischen Tipps zur Vermeidung von Fehlbefunden finden Sie umfangreiche Informationen zur sonographischen Diagnostik benigner und maligner urologischer Erkrankungen. Auf Normbefunde und Normvarianten wird zum besseren Verständnis gezielt eingegangen. Aktuelle Forschungsergebnisse und differenzialdiagnostische Tabellen zur schnellen Kontrolle der gestellten Diagnose runden das Werk ab. Die Auswahl der Bilder folgte höchsten Qualitätsansprüchen. Komplexe Befunde werden zusätzlich durch aussagekräftige Graphiken veranschaulicht. „Ultraschall in der Urologie“, ein unverzichtbarer Begleiter für alle, die sich mit der sonographischen Diagnostik des Urogenitaltrakts befassen. 